\title{
On a nonlocal 1-D initial value problem for a singular fractional-order parabolic equation with Bessel operator
}

\author{
Said Mesloub ${ }^{1 *}$ and Imed Bachar
}

"Correspondence:

mesloub@ksu.edu.sa

'Mathematics Department, College

of Science, King Saud University,

Riyadh, Saudi Arabia

\begin{abstract}
In this paper, we obtain some results of the existence and uniqueness of a generalized solution for a singular fractional initial boundary value problem in the Caputo sense subject to Neumann and weighted integral conditions. We show that a priori estimate or energy inequality methods can be successfully applied to obtaining a priori estimates for the solution of initial fractional boundary problems as in the classical case. The obtained results will contribute in the development of the functional analysis method and enrich the existing nonextensive literature on the nonlocal fractional mixed problems in the Caputo sense.
\end{abstract}

MSC: 35D35; 35L20

Keywords: Solvability of the problem; Weighted integral conditions; Fractional differential equation; Initial boundary value problem

\section{Introduction}

The one-dimensional fractional-order diffusion heat equation has become a real model for all linear and nonlinear fractional and nonfractional partial differential equations of parabolic type $[5,8,10,18,19]$. Although mathematical models in two and three dimensions are of great significance for applications, the majority of recent papers are devoted to the fractional-order diffusion equations in the one-dimensional case. Papers dealing with the multidimensional fractional diffusion equations are still not numerous. For fractional parabolic equations, we interpret physically the fractional derivative appearing in the equation as the degree of memory in the diffusing material [9]. Many authors have studied analytically and numerically various models of time-fractional differential equations; see, for example, $[2-4,7,13,20]$.

Many physical phenomena can be modeled in terms of local and nonlocal initial boundary value problems where the classical time and space derivatives are present, but, unfortunately, many others cannot be modeled by such problems. Different methods have been used to solve fractional diffusion equations. We can cite, for example, the works [11, 17].

In this paper, we apply the traditional functional analysis method, the so-called energy inequality method based mainly on some a priori bounds and on the density of the range of the operator generated by the considered problem for a fractional singular equation with Bessel operator and Caputo fractional derivative of order $0<\alpha<1$ (see [6]).

(c) The Author(s) 2019. This article is distributed under the terms of the Creative Commons Attribution 4.0 International License (http://creativecommons.org/licenses/by/4.0/), which permits unrestricted use, distribution, and reproduction in any medium, provided you give appropriate credit to the original author(s) and the source, provide a link to the Creative Commons license, and indicate if changes were made. 
In the literature, there are very many papers using the functional analysis method for the proof of the well-posedness of mixed problems (having local or nonlocal boundary conditions) in the classical sense, such as $[14,15,21]$, but in the fractional case, there are only few papers using the previous method to prove their well-posedness. Therefore our work can be considered as a contribution to the development of the functional analysis method used to prove the well-posedness of mixed problems with fractional order. We should like also to mention that the positivity of the fractional derivative operator helps us to obtain a priori bounds for solutions of certain classes of fractional initial and boundary value problems.

This paper is organized as follows: In Sect. 2, we set and pose the problem and give different types of fractional derivatives used in the paper. In Sect. 3, we introduce some function spaces, give some useful tools, and write the given problem in operator form. In Sect. 4, by choosing an appropriate functional differential operator multiplier we establish an a priori estimate, from which we deduce the uniqueness of the solution and its dependence on the given data of the posed problem. In Sect. 5, we prove the main result concerning the solvability of the given problem. With some modifications in the classical method (energy inequality method) used for classical equations, we could show that the range of the operator generated by the studied problem is dense in the weighted Hilbert space $H=L_{x}^{2}(0,1) \times L_{x}^{2}(Q)$, where $Q=(0,1) \times(0, T), T<\infty$.

\section{Problem setting}

We consider the governing equation of Caputo's time fractional order subject to initial and boundary conditions of integral and Neumann types in the domain $Q=(0,1) \times(0, T)$, $T<\infty$. By $\partial_{t}^{\alpha} \theta$ we denote the Caputo time fractional derivative. This initial boundary value problem is nonlocal in time derivative and in one of the boundary conditions:

$$
\left\{\begin{array}{l}
\mathcal{L} \theta=\partial_{t}^{\alpha} \theta-\frac{1}{x} \frac{\partial}{\partial x}\left(x \theta_{x}\right)+Y(x, t) \theta=f(x, t), \quad 0<x<1,0<t<T, \\
l_{1} \theta=\theta(x, 0)=\omega(x), \quad x \in(0,1), \\
\int_{0}^{1} x \theta(x, t) d x=0, \quad \theta_{x}(1, t)=0, t \in(0, T),
\end{array}\right.
$$

The functions $Y(x, t)$ and $f(x, t)$ are given functions, which will be specified later.

The time fractional derivative of order $0<\alpha<1$ is taken in the Caputo sense. It is defined for a differentiable function by

$$
\begin{array}{ll}
\partial_{t}^{\alpha} \theta(x, t)=\frac{1}{\Gamma(1-\alpha)} \frac{d}{d t} \int_{0}^{t} \frac{\theta(x, \tau)-\theta(x, 0)}{(t-\tau)^{\alpha}} d \tau, & t>0 \text { (LCFD), } \\
\partial_{T}^{\alpha} \theta(x, t)=\frac{1}{\Gamma(1-\alpha)} \frac{d}{d t} \int_{t}^{T} \frac{\theta(x, \tau)-\theta(x, 0)}{(t-\tau)^{\alpha}} d \tau, & t>0 \text { (RFFD), }
\end{array}
$$

or, equivalently,

$$
\begin{array}{ll}
\partial_{t}^{\alpha} \theta(x, t)=\frac{1}{\Gamma(1-\alpha)} \int_{0}^{t} \frac{\theta^{\prime}(x, \tau)}{(t-\tau)^{\alpha}} d \tau, & t>0(\mathrm{LCFD}), \\
\partial_{T}^{\alpha} \theta(x, t)=\frac{1}{\Gamma(1-\alpha)} \int_{t}^{T} \frac{\theta^{\prime}(x, \tau)}{(t-\tau)^{\alpha}} d \tau, & t<T(\mathrm{RCFD}),
\end{array}
$$

where $\Gamma$ is the gamma function. 
We also need to use the Riemann-Liouville integral of order $0<\alpha<1$ defined by

$$
D_{t}^{-\alpha} V(t)=\frac{1}{\Gamma(\alpha)} \int_{0}^{t} \frac{V(\tau)}{(t-\tau)^{1-\alpha}} d \tau
$$

For different properties of the Caputo fractional derivative, we refer the reader to [12, 16] and the references therein.

\section{Preliminaries}

We need the following function spaces and tools. We denote by $C^{2,1}(\bar{Q})$ the set of functions that, together with their partial derivatives of orders 2 and 1 in $x$ and $t$, are continuous on $\bar{Q}$, by $C^{m}(0, T)$ the space of $m$-fold differentiable functions, and by $C_{0}^{\infty}(0, T)$ the space of infinitely differentiable functions having their support in $(0, T)$. We use the usual $L^{2}(0, T)$ space of measurable square-integrable functions on $(0, T)$.

Lemma 3.1 ([1]) For any absolutely continuous function $\beta(s)$ on the interval $[0, T]$, we have the inequality

$$
\beta(s) \partial_{s}^{\alpha} \beta(s) \geq \frac{1}{2} \partial_{s}^{\alpha} \beta^{2}(s), \quad 0<\alpha<1 .
$$

Lemma $3.2([1])$ Let a nonnegative absolutely continuous function $\mathcal{R}(s)$ satisfy the inequality

$$
\partial_{t}^{\alpha} \mathcal{R}(s) \leq c_{1} \mathcal{R}(s)+c_{2}(s), \quad 0<\alpha<1,
$$

for almost all $s \in[0, T]$, where $c_{1}$ is a positive constant, and $c_{2}(s)$ is an integrable nonnegative function on $[0, T]$. Then

$$
\mathcal{R}(s) \leq \mathcal{R}(0) E_{\alpha}\left(c_{1} s^{\alpha}\right)+\Gamma(\alpha) E_{\alpha, \alpha}\left(c_{1} s^{\alpha}\right) D_{t}^{-\alpha} c_{2}(s),
$$

where

$$
E_{\alpha}(x)=\sum_{n=0}^{\infty} \frac{x^{n}}{\Gamma(\alpha n+1)} \quad \text { and } \quad E_{\alpha, \mu}(x)=\sum_{n=0}^{\infty} \frac{x^{n}}{\Gamma(\alpha n+\mu)}
$$

are the Mittag-Leffler functions.

Young's inequality with $\varepsilon$ : For any $\varepsilon>0$, we have the inequality

$$
a Y \leq \frac{1}{p}|\varepsilon a|^{p}+\frac{p-1}{p}\left|\frac{Y}{\varepsilon}\right|^{\frac{p}{p-1}}, \quad a, b \in \mathbb{R}, p>1,
$$

which is the generalization of the Cauchy inequality with $\varepsilon$ :

$$
a Y \leq \frac{\varepsilon}{2} a^{2}+\frac{1}{2 \varepsilon} Y^{2}, \quad \varepsilon>0
$$

where $a$ and $Y$ are nonnegative numbers. 
Poincaré-type inequalities [14]:

$$
\begin{aligned}
\left\|\mathcal{I}_{x}(\xi \theta)\right\|_{L^{2}(0, b)}^{2} & \leq \frac{b^{3}}{2}\|\theta\|_{L_{x}^{2}(0, b)}^{2}, \\
\left\|\mathcal{I}_{x}^{2}(\xi \theta)\right\|_{L^{2}(0, b)}^{2} & \leq \frac{b^{2}}{2}\left\|\mathcal{I}_{x}(\xi \theta)\right\|_{L^{2}(0, b)}^{2},
\end{aligned}
$$

where

$$
\mathcal{I}_{x}(U)=\int_{0}^{x} U(\xi, t) d \xi, \quad \mathcal{I}_{x}^{2}(U)=\int_{0}^{x} \int_{0}^{\xi} U(\eta, t) d \eta d \xi .
$$

To establish the existence and uniqueness of the solution of problem (2.1), we write it in an equivalent operator form.

The solution of problem (2.1) can be regarded as the solution of the operator equation

$$
\mathcal{M} \theta=\mathcal{F},
$$

where $\mathcal{M}=\left(\mathcal{L}, l_{1}\right)$, and the operator $\mathcal{M}$ acts from $\mathcal{S}$ to $H$ with domain of definition

$$
D(\mathcal{M})=\left\{\begin{array}{l}
\theta \in L_{x}^{2}(Q): \theta_{x}, \theta_{x x}, \partial_{t}^{\alpha} \theta \in L_{x}^{2}(Q), \\
\int_{0}^{1} x \theta(x, t) d x=0, \quad t \in(0, T), \\
\theta_{x}(1, t)=0, \quad t \in(0, T)
\end{array}\right.
$$

where $\mathcal{S}$ is a Banach space of functions $\theta$ endowed by the finite norm

$$
\begin{aligned}
\|\theta\|_{\mathcal{S}}^{2}= & \sup _{0 \leq t \leq T}\left(D^{\alpha-1}\left\|\mathcal{I}_{x}(\xi \theta)\right\|_{L^{2}(0,1)}^{2}+D^{\alpha-1}\|\theta\|_{L_{x}^{2}(0,1)}^{2}\right) \\
& +\int_{0}^{T}\left\|\theta_{x}\right\|_{L_{x}^{2}(0,1)}^{2} d t
\end{aligned}
$$

and $H$ is the weighted Hilbert space $L_{x}^{2}(Q) \times L_{x}^{2}(0,1)$ consisting of vector-valued functions $\mathcal{F}=(f, \omega)$ with finite norm

$$
\|\mathcal{F}\|_{H}^{2}=\|\omega\|_{L_{x}^{2}(0,1)}^{2}+\|f\|_{L_{x}^{2}(Q)}^{2} .
$$

\section{A priori estimate for the solution and its consequences}

We establish an a priori bound for the solution of problem (2.1), from which we deduce its uniqueness.

Theorem 4.1 Suppose that the function $Y$ satisfies

$$
\text { (i) } \quad Y(x, t) \geq C_{0}, \quad \text { (ii) } \quad Y_{x x}(x, t) \leq C_{1}, \quad(x, t) \in Q \text {, }
$$

where $C_{0}$ and $C_{1}$ are positive constants, and $f \in L_{x}^{2}(Q)$. Then there exists a positive constant $\mu$ such that the following a priori estimate holds:

$$
\sup _{0 \leq t \leq T}\left(D^{\alpha-1}\left\|\mathcal{I}_{x}(\xi \theta)\right\|_{L^{2}(0,1)}^{2}+D^{\alpha-1}\|\theta\|_{L_{x}^{2}(0,1)}^{2}\right)+\int_{0}^{T}\left\|\theta_{x}\right\|_{L_{x}^{2}(0,1)}^{2} d t
$$




$$
\leq \mu\left(\|\omega\|_{L_{x}^{2}(0,1)}^{2}+\|f\|_{L_{x}^{2}(Q)}^{2}\right)
$$

for all $\theta \in D(\mathcal{M})$, where $\mu=\mu(\delta, \sigma, d)$ is given by

$$
\mu=\delta \max \left(\frac{3}{2}, 1+\frac{T^{\alpha}}{\Gamma(1+\alpha)}\right),
$$

and $\delta, \sigma, d$ are respectively given by (4.29), (4.24), and (4.22).

Proof Consider the identity

$$
\begin{aligned}
& \left(\partial_{t}^{\alpha} \theta-\frac{1}{x} \frac{\partial}{\partial x}\left(x \frac{\partial \theta}{\partial x}\right)+Y(x, t) \theta, x \theta-x \mathcal{I}_{x}^{2}(\xi \theta)\right)_{L^{2}(0,1)} \\
& =\left(f, x \theta-x \mathcal{I}_{x}^{2}(\xi \theta)\right)_{L^{2}(0,1)} .
\end{aligned}
$$

Under the boundary and initial conditions in (2.1), the terms of the left-hand side of (4.4) give

$$
\begin{aligned}
& -\left(\partial_{t}^{\alpha} \theta, x \mathcal{I}_{x}^{2}(\xi \theta)\right)_{L^{2}(0,1)} \\
& =-\int_{0}^{1} x \partial_{t}^{\alpha} \theta\left(\mathcal{I}_{x}^{2}(\xi \theta)\right) d x \\
& =-\left.\left(\partial_{t}^{\alpha} \int_{0}^{x} \xi \theta d \xi\right)\left(\mathcal{I}_{x}^{2}(\xi \theta)\right)\right|_{0} ^{1}+\int_{0}^{1}\left(\partial_{t}^{\alpha} \mathcal{I}_{x}(\xi \theta)\right)\left(\mathcal{I}_{x}(\xi \theta)\right) d x \\
& =\int_{0}^{1}\left(\partial_{t}^{\alpha} \mathcal{I}_{x}(\xi \theta)\right)\left(\mathcal{I}_{x}(\xi \theta)\right) d x, \\
& \left(\frac{\partial}{\partial x}\left(x \frac{\partial \theta}{\partial x}\right), \mathcal{I}_{x}^{2}(\xi \theta)\right)_{L^{2}(0,1)}=-\int_{0}^{1} x \frac{\partial \theta}{\partial x} \mathcal{I}_{x}(\xi \theta) d x, \\
& -\left(Y(x, t) \theta, x \mathcal{I}_{x}^{2}(\xi \theta)\right)_{L^{2}(0,1)} \\
& =\int_{0}^{1} \frac{\partial Y(x, t)}{\partial x}\left(\mathcal{I}_{x}(\xi \theta)\right)\left(\mathcal{I}_{x}^{2}(\xi \theta)\right) d x+\int_{0}^{1} Y(x, t)\left(\mathcal{I}_{x}(\xi \theta)\right)^{2} d x \\
& =\int_{0}^{1} Y(x, t)\left(\mathcal{I}_{x}(\xi \theta)\right)^{2} d x-\frac{1}{2} \int_{0}^{1} \frac{\partial^{2} Y(x, t)}{\partial x^{2}}\left(\mathcal{I}_{x}^{2}(\xi \theta)\right)^{2} d x, \\
& \left(\partial_{t}^{\alpha} \theta, x \theta\right)_{L^{2}(0,1)}=\int_{0}^{1} x \theta \partial_{t}^{\alpha} \theta d x, \\
& -\left(\frac{\partial}{\partial x}\left(x \frac{\partial \theta}{\partial x}\right), \theta\right)_{L^{2}(0,1)}=\int_{0}^{1} x \theta_{x}^{2} d x \\
& (Y(x, t) \theta, x \theta)_{L^{2}(0,1)}=\int_{0}^{1} x Y(x, t) \theta^{2} d x .
\end{aligned}
$$

Substitution of equalities (4.5)-(4.10) into (4.4) yields

$$
\begin{aligned}
& \int_{0}^{1}\left(\partial_{t}^{\alpha} \mathcal{I}_{x}(\xi \theta)\right)\left(\mathcal{I}_{x}(\xi \theta)\right) d x+\int_{0}^{1} x \theta \partial_{t}^{\alpha} \theta d x \\
& \quad+\int_{0}^{1} x Y(x, t) \theta^{2} d x+\int_{0}^{1} Y\left((x, t)\left(\mathcal{I}_{x}(\xi \theta)\right)^{2} d x+\int_{0}^{1} x \theta_{x}^{2} d x\right.
\end{aligned}
$$




$$
\begin{aligned}
= & \frac{1}{2} \int_{0}^{1} \frac{\partial^{2} Y(x, t)}{\partial x^{2}}\left(\mathcal{I}_{x}^{2}(\xi \theta)\right)^{2} d x+\int_{0}^{1} x \frac{\partial \theta}{\partial x} \mathcal{I}_{x}(\xi \theta) d x \\
& +\left(\mathcal{I}_{x}(\xi f), \mathcal{I}_{x}(\xi \theta)\right)_{L^{2}(0,1)}+(x \theta, f)_{L^{2}(0,1)} .
\end{aligned}
$$

To estimate the first and second terms on the left-hand side of (4.11), we use Lemma 3.1 to obtain

$$
\begin{aligned}
& \int_{0}^{1}\left(\partial_{t}^{\alpha} \mathcal{I}_{x}(\xi \theta)\right)\left(\mathcal{I}_{x}(\xi \theta)\right) d x \\
& \geq \frac{1}{2} \int_{0}^{1} \partial_{t}^{\alpha}\left(\mathcal{I}_{x}(\xi \theta)\right)^{2} d x \\
& \int_{0}^{1} x \theta \partial_{t}^{\alpha} \theta d x \geq \frac{1}{2} \int_{0}^{1} \partial_{t}^{\alpha}\left(x \theta^{2}\right) d x
\end{aligned}
$$

Conditions (4.1) and inequality (3.8) lead to

$$
\begin{aligned}
& \int_{0}^{1} \frac{\partial^{2} Y(x, t)}{\partial x^{2}}\left(\mathcal{I}_{x}^{2}(\xi \theta)\right)^{2} d x \\
& \quad \leq C_{1} \int_{0}^{1}\left(\mathcal{I}_{x}^{2}(\xi \theta)\right)^{2} d x \leq \frac{C_{1}}{2} \int_{0}^{1}\left(\mathcal{I}_{x}(\xi \theta)\right)^{2} d x \\
& \int_{0}^{1} Y(x, t)\left(\mathcal{I}_{x}(\xi \theta)\right)^{2} d x \geq C_{0} \int_{0}^{1}\left(\mathcal{I}_{x}(\xi \theta)\right)^{2} d x \\
& \int_{0}^{1} x Y(x, t) \theta^{2} d x \geq C_{0} \int_{0}^{1} x \theta^{2} d x
\end{aligned}
$$

The terms on the right-hand side of (4.11) can be estimated in the following way:

$$
\begin{aligned}
& \left(\mathcal{I}_{x}(\xi f), \mathcal{I}_{x}(\xi \theta)\right)_{L^{2}(0,1)} \\
& \quad \leq \frac{\varepsilon_{1}}{2} \int_{0}^{1}\left(\mathcal{I}_{x}(\xi \theta)\right)^{2} d x+\frac{1}{4 \varepsilon_{1}} \int_{0}^{1} x f^{2} d x, \\
& \int_{0}^{1} x \theta_{x} \mathcal{I}_{x}(\xi \theta) d x \\
& \quad \leq \frac{\varepsilon_{2}}{2} \int_{0}^{1}\left(\mathcal{I}_{x}(\xi \theta)\right)^{2} d x+\frac{1}{4 \varepsilon_{2}} \int_{0}^{1} x \theta_{x}^{2} d x, \\
& (x \theta, f)_{L^{2}(0,1)} \leq \frac{1}{2} \int_{0}^{1} x \theta^{2} d x+\frac{1}{2} \int_{0}^{1} x f^{2} d x .
\end{aligned}
$$

Combination of (4.11)-(4.19) yields

$$
\begin{gathered}
\int_{0}^{1} \partial_{t}^{\alpha}\left(\mathcal{I}_{x}(\xi \theta)\right)^{2} d x+\frac{1}{2} \int_{0}^{1} \partial_{t}^{\alpha}\left(x \theta^{2}\right) d x+\int_{0}^{1} x \theta_{x}^{2} d x \\
+C_{0} \int_{0}^{1}\left(\mathcal{I}_{x}(\xi \theta)\right)^{2} d x+C_{0} \int_{0}^{1} x \theta^{2} d x \\
\leq \frac{C_{1}}{2} \int_{0}^{1}\left(\mathcal{I}_{x}(\xi \theta)\right)^{2} d x+\frac{\varepsilon_{1}}{2} \int_{0}^{1}\left(\mathcal{I}_{x}(\xi \theta)\right)^{2} d x
\end{gathered}
$$




$$
\begin{aligned}
& +\frac{1}{4 \varepsilon_{1}} \int_{0}^{1} x f^{2} d x+\frac{\varepsilon_{2}}{2} \int_{0}^{1}\left(\mathcal{I}_{x}(\xi \theta)\right)^{2} d x+\frac{1}{4 \varepsilon_{2}} \int_{0}^{1} x \theta_{x}^{2} d x \\
& +\frac{\varepsilon_{3}}{2} \int_{0}^{1} x \theta^{2} d x+\frac{1}{2 \varepsilon_{3}} \int_{0}^{1} x f^{2} d x .
\end{aligned}
$$

By choosing $\varepsilon_{1}=2 C_{0}, \varepsilon_{2}=\frac{1}{2}$, and $\varepsilon_{3}=3 C_{0}(4.20)$ becomes

$$
\begin{aligned}
& \int_{0}^{1} \partial_{t}^{\alpha}\left(\mathcal{I}_{x}(\xi \theta)\right)^{2} d x+\int_{0}^{1} \partial_{t}^{\alpha}\left(x \theta^{2}\right) d x+\int_{0}^{1} x \theta_{x}^{2} d x \\
& \quad \leq d\left(\int_{0}^{1}\left(\mathcal{I}_{x}(\xi \theta)\right)^{2} d x+\int_{0}^{1} x \theta^{2} d x+\int_{0}^{1} x f^{2} d x\right),
\end{aligned}
$$

where

$$
d=\frac{\max \left(\frac{C_{1}}{2}+\frac{1}{4}, \frac{C_{0}}{2}, \frac{5 C_{0}}{2}, \frac{1}{2}\right)}{\min \left(\frac{C_{0}}{2}, \frac{1}{2}\right)} .
$$

Replacing $t$ by $\tau$ and integrating both sides of (4.21) with respect to $\tau$ from 0 to $t$, we obtain

$$
\begin{aligned}
D^{\alpha-1} & \int_{0}^{1}\left(\mathcal{I}_{x}(\xi \theta)\right)^{2} d x+D^{\alpha-1} \int_{0}^{1} x \theta^{2} d x+\int_{0}^{t} \int_{0}^{1} x \theta_{x}^{2} d x d \tau \\
\leq & \sigma\left(\int_{0}^{t} \int_{0}^{1}\left(\mathcal{I}_{x}(\xi \theta)\right)^{2} d x d \tau+\int_{0}^{t} \int_{0}^{1} x \theta^{2} d x d \tau+\int_{0}^{t} \int_{0}^{1} x f^{2} d x d \tau\right. \\
& \left.+\int_{0}^{1}\left(\mathcal{I}_{x}(\xi \omega)\right)^{2} d x+\int_{0}^{1} x \omega^{2} d x\right)
\end{aligned}
$$

where

$$
\sigma=\max \left\{d, \frac{T^{1-\alpha}}{(1-\alpha) \Gamma(1-\alpha)}\right\} .
$$

Dropping the last term on the left-hand side of (4.23), applying Lemma 3.2, and setting

$$
\begin{aligned}
& \mathcal{R}(t)=\int_{0}^{t} \int_{0}^{1}\left(\mathcal{I}_{x}(\xi \theta)\right)^{2} d x d \tau+\int_{0}^{t} \int_{0}^{1} x \theta^{2} d x, \quad \mathcal{R}(0)=0 \\
& \partial_{t}^{\alpha} \mathcal{R}(t)=D^{\alpha-1}\left(\mathcal{I}_{x}(\xi \theta)\right)^{2} d x+D^{\alpha-1} \int_{0}^{1} x \theta^{2} d x
\end{aligned}
$$

we obtain

$$
\begin{aligned}
\int_{0}^{t} & \int_{0}^{1}\left(\mathcal{I}_{x}(\xi \theta)\right)^{2} d x d \tau+\int_{0}^{t} \int_{0}^{1} x \theta^{2} d x \\
\leq & \Gamma(\alpha) E_{\alpha, \alpha}\left(d t^{\alpha}\right) \cdot\left(D_{t}^{-\alpha-1} \int_{0}^{1} x f^{2} d x+\int_{0}^{1}\left(\mathcal{I}_{x} \xi \omega\right)^{2} d x\right. \\
& \left.+\int_{0}^{1} x \omega^{2} d x\right) .
\end{aligned}
$$

On the light of (4.27) and (3.7), inequality (4.23) becomes

$$
D^{\alpha-1}\left\|\mathcal{I}_{x}(\xi \theta)\right\|_{L^{2}(0,1)}^{2}+D^{\alpha-1}\|\theta\|_{L_{x}^{2}(0,1)}^{2}+\int_{0}^{t}\left\|\theta_{x}\right\|_{L_{x}^{2}(0,1)}^{2} d \tau
$$




$$
\begin{aligned}
\leq & \delta\left(D_{t}^{-1-\alpha}\|f\|_{L_{x}^{2}(0,1)}^{2}+\left\|\mathcal{I}_{x}(\xi \omega)\right\|_{L^{2}(0,1)}^{2}\right. \\
& \left.+\|\omega\|_{L_{x}^{2}(0,1)}^{2}+\int_{0}^{t}\|f\|_{L_{x}^{2}(0,1)}^{2} d \tau\right) \\
\leq & \delta\left(D_{t}^{-1-\alpha}\|f\|_{L_{x}^{2}(0,1)}^{2}+\|\omega\|_{L_{x}^{2}(0,1)}^{2}+\int_{0}^{t}\|f\|_{L_{x}^{2}(0,1)}^{2} d \tau\right),
\end{aligned}
$$

where

$$
\delta=\max \left\{\sigma \Gamma(\alpha) E_{\alpha, \alpha}\left(d t^{\alpha}\right), \sigma\right\} .
$$

It is easy to see that

$$
D_{t}^{-\alpha-1}\|f\|_{L_{x}^{2}(0,1)}^{2} \leq \frac{T^{\beta}}{\Gamma(1+\beta)} \int_{0}^{T}\|f\|_{L_{x}^{2}(0,1)}^{2} d \tau .
$$

Inequalities (4.28) and (4.30) yield

$$
\begin{aligned}
& D^{\alpha-1}\left\|\mathcal{I}_{x}(\xi \theta)\right\|_{L^{2}(0,1)}^{2}+D^{\alpha-1}\|\theta\|_{L_{x}^{2}(0,1)}^{2}+\int_{0}^{t}\left\|\theta_{x}\right\|_{L_{x}^{2}(0,1)}^{2} d \tau \\
& \quad \leq \mu\left(\|\omega\|_{L_{x}^{2}(0,1)}^{2}+\int_{0}^{T}\|f\|_{L_{x}^{2}(0,1)}^{2} d \tau\right),
\end{aligned}
$$

where

$$
\mu=\delta \max \left(\frac{3}{2}, 1+\frac{T^{\alpha}}{\Gamma(1+\alpha)}\right) .
$$

Now since the right-hand side of (4.31) does not depend on $t$, the a priori estimate (4.2) follows by taking the upper bound for both sides with respect to $t$ over $[0, T]$. Note that the uniqueness and continuous dependence of the solution on the data of problem (2.1) follows from the a priori bound (4.2).

\section{Existence of solution}

The a priori estimate (4.2) shows that the unbounded operator $\mathcal{M}$ has an inverse $\mathcal{M}^{-1}$ : $\mathcal{R}(\mathcal{M}) \rightarrow \mathcal{S}$. Since $\mathcal{R}(\mathcal{M})$ is a subset of $H$, we can construct its closure $\overline{\mathcal{M}}$ so that estimate (4.2) holds for this extension and $\mathcal{R}(\overline{\mathcal{M}})$ coincides with the whole space $H$. Hence we have the following:

Corollary 5.1 The operator $\mathcal{M}: \mathcal{S} \rightarrow H$ admits a closure (proof is similar to that in [14]. Estimate (4.2) can be then extended to

$$
\begin{aligned}
& \sup _{0 \leq t \leq T}\left(D^{\alpha-1}\left\|\mathcal{I}_{x}(\xi \theta)\right\|_{L^{2}(0,1)}^{2}+D^{\alpha-1}\|\theta\|_{L_{x}^{2}(0,1)}^{2}\right)+\int_{0}^{T}\left\|\theta_{x}\right\|_{L_{x}^{2}(0,1)}^{2} d t \\
& \quad \leq \mu\left(\|\omega\|_{L_{x}^{2}(0,1)}^{2}+\|f\|_{L_{x}^{2}(Q)}^{2}\right)
\end{aligned}
$$

for all $\theta \in D(\overline{\mathcal{M}})$. 
It follows from (5.1) that the strong solution of problem (2.1) is unique, that is, $\overline{\mathcal{M}} \theta=H$. From estimate (5.1) we also deduce the following:

Corollary 5.2 $\mathcal{R}(\overline{\mathcal{M}})$ is a closed subset in $H, \mathcal{R}(\mathcal{M})=\mathcal{R}(\overline{\mathcal{M}})$, and $\overline{\mathcal{M}}^{-1}=\overline{\mathcal{M}}^{-1}$.

We are now ready to give the result on the existence of the solution of problem (2.1).

Theorem 5.3 Suppose that the conditions of Theorem 4.1 are satisfied. Then for all $\mathcal{F}=$ $(f, \omega) \in H$, there exists a unique strong solution $\theta=\overline{\mathcal{M}}^{-1} \mathcal{F}=\overline{\mathcal{M}^{-1}} \mathcal{F}$ of problem (2.1).

Proof Estimate (5.1) asserts that if a strong solution of (2.1) exits, then it is unique and depends continuously on the data. Corollary 5.2 says that to prove that problem (2.1) admits a strong solution for any $\mathcal{F}=(f, \omega) \in H$, it suffices to show that the closure of the range of the operator $\mathcal{M}$ is dense in $H$. To establish the existence of the strong solution of problem (2.1), we use a density argument, that is, we show that the range $\mathcal{R}(\mathcal{M})$ of the operator $\mathcal{M}$, is dense in the space $H$ for every element $\theta$ in the Banach space $\mathcal{S}$. For this, we consider the following particular case of density.

Theorem 5.4 Suppose that the conditions of Theorem 4.1 are satisfied. Suppose that for all functions $\theta \in \mathcal{D}(\mathcal{M})$ such that $l_{1} \theta=\theta(x, 0)=0$ and for some function $\psi \in L^{2}(Q)$, we have

$$
\int_{0}^{T}(\mathcal{L} \theta, \psi)_{L_{x}^{2}(0,1)} d t=0
$$

Then $\psi$ vanishes a.e. in $Q$.

Proof Identity (5.2) is equivalent to

$$
\int_{0}^{T}\left(\partial_{t}^{\alpha} \theta-\frac{1}{x} \frac{\partial}{\partial x}\left(x \frac{\partial \theta}{\partial x}\right)+Y(x, t) \theta, \psi\right)_{L_{x}^{2}(0,1)} d t=0
$$

Assume that a function $\gamma(x, t)$ satisfies the conditions boundary and initial conditions in (2.1) and that $\gamma, \gamma_{x}$, and $\frac{\partial}{\partial x}\left(x \int_{0}^{t} \gamma(x, s) d s\right) \in L^{2}\left(Q_{t}\right)$. We then set

$$
\theta(x, t)=\int_{0}^{t} \gamma(x, s) d s
$$

Equation (5.3) then becomes

$$
\begin{aligned}
& \int_{0}^{T}\left\{\partial_{t}^{\alpha}\left(\int_{0}^{t} \gamma(x, s) d s\right)-\frac{1}{x} \frac{\partial}{\partial x}\left(\int_{0}^{t} x \gamma_{x}(x, s) d s\right)\right. \\
& \left.\left.\quad+Y(x, t)\left(\int_{0}^{t} \gamma(x, s) d s\right), \psi\right)_{L_{x}^{2}(0,1)}\right\} d t \\
& =0 .
\end{aligned}
$$

We now introduce the function

$$
\psi(x, t)=\int_{0}^{t} \gamma(x, s) d s-\mathcal{I}_{x}^{2}\left(\xi \int_{0}^{t} \gamma(\xi, s) d s\right) .
$$


Mesloub and Bachar Advances in Difference Equations

(2019) 2019:254

Page 10 of 14

Equation (5.5) then reduces to

$$
\begin{aligned}
\int_{0}^{T} & \left(\partial_{t}^{\alpha}\left(\int_{0}^{t} \gamma(x, s) d s\right), x \int_{0}^{t} \gamma(x, s) d s-x \mathcal{I}_{x}^{2}\left(\xi \int_{0}^{t} \gamma(\xi, s) d s\right)\right)_{L^{2}(0,1)} d t \\
& +\int_{0}^{T}\left(\frac{\partial}{\partial x}\left(\int_{0}^{t} x \gamma_{x}(x, s) d s\right),-\int_{0}^{t} \gamma(x, s) d s+\mathcal{I}_{x}^{2}\left(\xi \int_{0}^{t} \gamma(\xi, s) d s\right)\right)_{L^{2}(0,1)} d t \\
& -\int_{0}^{T}\left(Y(x, t)\left(\int_{0}^{t} \gamma(x, s) d s\right),-x \int_{0}^{t} \gamma(x, s) d s+x \mathcal{I}_{x}^{2}\left(\xi \int_{0}^{t} \gamma(\xi, s) d s\right)\right)_{L^{2}(0,1)} d t \\
\quad & 0 .
\end{aligned}
$$

Recall that the function $\gamma$ satisfies boundary conditions in (2.1). Then computing the inner products in (5.7), we have

$$
\begin{aligned}
& -\left(\partial_{t}^{\alpha}\left(\int_{0}^{t} \gamma(x, s) d s\right), x \mathcal{I}_{x}^{2}\left(\xi \int_{0}^{t} \gamma(\xi, s) d s\right)\right)_{L^{2}(0,1)} \\
& =\left(\partial_{t}^{\alpha}\left(\mathcal{I}_{x}\left(\xi \int_{0}^{t} \gamma(\xi, s) d s\right)\right), \mathcal{I}_{x}\left(\xi \int_{0}^{t} \gamma(\xi, s) d s\right)\right)_{L^{2}(0,1)} \\
& \geq \frac{1}{2} \int_{0}^{1} \partial_{t}^{\alpha}\left(\mathcal{I}_{x}\left(\xi \int_{0}^{t} \gamma(\xi, s) d s\right)\right)^{2} d x \\
& =\frac{1}{2} \partial_{t}^{\alpha}\left\|\mathcal{I}_{x} \int_{0}^{t} \xi \gamma(\xi, s) d s\right\|_{L^{2}(0,1)}^{2}, \\
& \left(\frac{\partial}{\partial x}\left(\int_{0}^{t} x \gamma_{x}(x, s) d s\right), \mathcal{I}_{x}^{2}\left(\xi \int_{0}^{t} \gamma(\xi, s) d s\right)\right)_{L^{2}(0,1)} \\
& =-\left(\int_{0}^{t} x \gamma_{x}(x, s) d s, \mathcal{I}_{x}\left(\xi \int_{0}^{t} \gamma(\xi, s) d s\right)\right)_{L^{2}(0,1)}, \\
& -\left(Y(x, t)\left(\int_{0}^{t} \gamma(x, s) d s\right), x \mathcal{I}_{x}^{2}\left(\xi \int_{0}^{t} \gamma(\xi, s) d s\right)\right)_{L^{2}(0,1)} \\
& =\left(\frac{\partial Y(x, t)}{\partial x} \mathcal{I}_{x}\left(\xi \int_{0}^{t} \gamma(\xi, s) d s\right), \mathcal{I}_{x}^{2}\left(\xi \int_{0}^{t} \gamma(\xi, s) d s\right)\right)_{L^{2}(0,1)} \\
& +\int_{0}^{1} Y(x, t)\left(\mathcal{I}_{x}\left(\xi \int_{0}^{t} \gamma(\xi, s) d s\right)\right)^{2} d x \\
& =\int_{0}^{1} Y(x, t)\left(\mathcal{I}_{x}\left(\xi \int_{0}^{t} \gamma(\xi, s) d s\right)\right)^{2} d x \\
& -\int_{0}^{1} \frac{\partial^{2} Y(x, t)}{\partial x^{2}}\left(\mathcal{I}_{x}^{2}\left(\xi \int_{0}^{t} \gamma(\xi, s) d s\right)\right)^{2} d x, \\
& -\left(\frac{\partial}{\partial x}\left(\int_{0}^{t} x \gamma_{x}(x, s) d s\right), \int_{0}^{t} \gamma(x, s) d s\right)_{L^{2}(0,1)} \\
& =\left\|\int_{0}^{t} \gamma_{x}(x, s) d s\right\|_{L_{x}^{2}(0,1)}^{2}, \\
& \left(Y(x, t)\left(\int_{0}^{t} \gamma(x, s) d s\right), x \int_{0}^{t} \gamma(x, s) d s\right)_{L^{2}(0,1)}
\end{aligned}
$$




$$
\begin{gathered}
\quad=\left\|\sqrt{Y(x, t)} \int_{0}^{t} \gamma(x, s) d s\right\|_{L_{x}^{2}(0,1)}^{2} \cdot \\
\left(\partial_{t}^{\alpha}\left(\int_{0}^{t} \gamma(x, s) d s\right), x \int_{0}^{t} \gamma(x, s) d s\right)_{L^{2}(0,1)} \\
\geq \frac{1}{2} \int_{0}^{1} x \partial_{t}^{\alpha}\left(\int_{0}^{t} \gamma(x, s) d s\right)^{2} d x \\
=\frac{1}{2} \partial_{t}^{\alpha}\left\|\int_{0}^{t} \gamma(x, s) d s\right\|_{L_{x}^{2}(0,1)}^{2} .
\end{gathered}
$$

Using (5.8)-(5.13) and the Cauchy $\varepsilon$-inequality, replacing $t$ by $\tau$, and integrating with respect to $\tau$ over $(0, t)$, we see that

$$
\begin{aligned}
& \frac{1}{2} D_{t}^{\alpha-1}\left\|\mathcal{I}_{x} \int_{0}^{t} \xi \gamma(\xi, s) d s\right\|_{L^{2}(0,1)}^{2}+\frac{1}{2} D_{t}^{\alpha-1}\left\|\int_{0}^{t} \gamma(x, s) d s\right\|_{L_{x}^{2}(0,1)}^{2} \\
& \quad+\int_{0}^{t} \int_{0}^{1} Y(x, \tau)\left(\mathcal{I}_{x}\left(\xi \int_{0}^{\tau} \gamma(\xi, s) d s\right)\right)^{2} d x d \tau+\int_{0}^{t}\left\|\int_{0}^{\tau} \gamma_{x}(x, s) d s\right\|_{L_{x}^{2}(0,1)}^{2} d \tau \\
& \quad+\int_{0}^{t}\left\|\sqrt{Y(x, \tau)} \int_{0}^{\tau} \gamma(x, s) d s\right\|_{L_{x}^{2}(0,1)}^{2} d \tau \\
& \leq \frac{1}{\varepsilon} \int_{0}^{t} \int_{0}^{1}\left(\mathcal{I}_{x}\left(\xi \int_{0}^{\tau} \gamma(\xi, s) d s\right)\right)^{2} d x d \tau+\frac{\varepsilon}{2} \int_{0}^{t}\left\|\int_{0}^{\tau} \gamma_{x}(x, s) d s\right\|_{L_{x}^{2}(0,1)}^{2} d \tau \\
& \quad+\int_{0}^{t} \int_{0}^{1} \frac{\partial^{2} Y(x, \tau)}{\partial x^{2}}\left(\mathcal{I}_{x}^{2}\left(\xi \int_{0}^{\tau} \gamma(\xi, s) d s\right)\right)^{2} d x d \tau .
\end{aligned}
$$

Choosing $\varepsilon=1$ and using inequalities (3.6) and (3.7) and conditions (4.1), we rewrite inequality (5.14) as

$$
\begin{aligned}
D_{t}^{\alpha-1} \| & \mathcal{I}_{x} \int_{0}^{t} \xi \gamma(\xi, s) d s\left\|_{L^{2}(0,1)}^{2}+D_{t}^{\alpha-1}\right\| \int_{0}^{t} \gamma(x, s) d s \|_{L_{x}^{2}(0,1)}^{2} \\
& +2 C_{0} \int_{0}^{t}\left\|\mathcal{I}_{x} \int_{0}^{\tau} \xi \gamma(x, s) d s\right\|_{L^{2}(0,1)}^{2} d \tau+\int_{0}^{t}\left\|\int_{0}^{\tau} \gamma_{x}(x, s) d s\right\|_{L_{x}^{2}(0,1)}^{2} d \tau \\
& +2 C_{0} \int_{0}^{t}\left\|\int_{0}^{\tau} \gamma(x, s) d s\right\|_{L_{x}^{2}(0,1)}^{2} d \tau \\
\leq & \int_{0}^{t}\left\|\int_{0}^{\tau} \xi \gamma(x, s) d s\right\|_{L_{x}^{2}(0,1)}^{2} d \tau+C_{1} \int_{0}^{t} \mathcal{I}_{x}\left\|\int_{0}^{\tau} \gamma(x, s) d s\right\|_{L_{x}^{2}(0,1)}^{2} d \tau .
\end{aligned}
$$

Discarding the last three terms on the left-hand side of (5.15), we obtain

$$
\begin{aligned}
& D_{t}^{\alpha-1}\left\|\mathcal{I}_{x} \int_{0}^{t} \xi \gamma(\xi, s) d s\right\|_{L^{2}(0,1)}^{2}+D_{t}^{\alpha-1}\left\|\int_{0}^{t} \gamma(x, s) d s\right\|_{L_{x}^{2}(0,1)}^{2} \\
& \quad \leq \mathcal{V}\left(\int_{0}^{t}\left\|\mathcal{I}_{x} \int_{0}^{\tau} \gamma(\xi, s) d s\right\|_{L^{2}(0,1)}^{2} d \tau+\int_{0}^{t}\left\|\int_{0}^{\tau} \gamma(\xi, s) d s\right\|_{L_{x}^{2}(0,1)}^{2} d \tau\right),
\end{aligned}
$$


where

$$
\mathcal{V}=\max \left(1, C_{1}\right)
$$

We can now apply Lemma 3.2 to (5.16) by taking

$$
\begin{aligned}
& \varphi(t)=\int_{0}^{t}\left\|\mathcal{I}_{x} \int_{0}^{\tau} \gamma(\xi, s) d s\right\|_{L^{2}(0,1)}^{2} d \tau+\int_{0}^{t}\left\|\int_{0}^{\tau} \gamma(\xi, s) d s\right\|_{L_{x}^{2}(0,1)}^{2} d \tau, \\
& \varphi(0)=0
\end{aligned}
$$

and

$$
\partial_{t}^{\alpha} \varphi(t)=D_{t}^{\alpha-1}\left\|\mathcal{I}_{x} \int_{0}^{t} \xi \gamma(\xi, s) d s\right\|_{L^{2}(0,1)}^{2}+D_{t}^{\alpha-1}\left\|\int_{0}^{t} \gamma(x, s) d s\right\|_{L_{x}^{2}(0,1)}^{2} .
$$

Then

$$
\begin{aligned}
& \int_{0}^{t}\left\|\mathcal{I}_{x} \int_{0}^{\tau} \gamma(\xi, s) d s\right\|_{L^{2}(0,1)}^{2} d \tau+\int_{0}^{t}\left\|\int_{0}^{\tau} \gamma(\xi, s) d s\right\|_{L_{x}^{2}(0,1)}^{2} d \tau \\
& \leq \varphi(0) E_{\alpha}\left(\mathcal{V} t^{\alpha}\right)+\Gamma(\alpha) E_{\alpha, \alpha}\left(\mathcal{V} t^{\alpha}\right) D_{t}^{-\alpha}(0)=0 .
\end{aligned}
$$

Inequality (5.19) implies that

$$
\int_{0}^{T}\left\|\mathcal{I}_{x} \int_{0}^{\tau} \gamma(\xi, s) d s\right\|_{L^{2}(0,1)}^{2} d \tau+\int_{0}^{T}\left\|\int_{0}^{\tau} \gamma(\xi, s) d s\right\|_{L_{x}^{2}(0,1)}^{2} d \tau \leq 0
$$

Then from (5.20) it follows that the function $\psi=\int_{0}^{t} \gamma(x, s) d s-\mathcal{I}_{x}^{2}\left(\xi \int_{0}^{t} \gamma(\xi, s) d s\right)$ is zero a.e. in $Q$.

To complete the proof of Theorem 5.3, assume that for $\left(\Psi, \omega_{1}\right) \in R(\mathcal{M})^{\perp}$, we have

$$
\int_{0}^{T}(\mathcal{L} \theta, \Psi)_{L_{x}^{2}(0,1)} d s+\left(l_{1} \theta, \omega_{1}\right)_{L_{x}^{2}(0,1)}=0
$$

Then we should show that $\Psi=0, \omega_{1}=0$. If we put $\theta \in D(\mathcal{M})$ satisfying condition $l_{1} \theta=$ $\theta(x, 0)=0$ into (5.20), we get

$$
\int_{0}^{T}(\mathcal{L} \theta, \Psi)_{L_{x}^{2}(0,1)} d s=0, \quad \theta \in D(\mathcal{M})
$$

By Theorem 5.4 equation (5.22) implies that $\Psi$ vanishes a.e. in $Q$. Then (5.21) becomes

$$
\left(l_{1} \theta, \omega_{1}\right)_{L_{x}^{2}(0,1)}=0, \quad \theta \in D(\mathcal{M}) .
$$

Since the range of the trace operator $l_{1}$ is dense in $L_{x}^{2}(0,1)$, from (5.22) we conclude that $\omega_{1}=0$, and Theorem 5.3 is proved.

We present the following example to illustrate our main results. 
Example In the considered problem (2.1), we set

$$
Y(x, t)=\left(x^{n}+1\right)\left(t^{v}+1\right), \quad n \in \mathbb{N}, v>0,
$$

and

$$
\begin{aligned}
f(x, t)= & \frac{\lambda(x) t^{1-\alpha}}{(1-\alpha) \Gamma(1-\alpha)}+\frac{2 \rho(x) t^{2-\alpha}}{(1-\alpha)(2-\alpha) \Gamma(1-\alpha)}+\frac{12}{5}\left(x^{-1}-2\right)(t+1) \\
& -t^{2}+\left(x^{n}+1\right)\left(t^{\nu}+1\right)\left[\lambda(t+1)+\rho t^{2}\right],
\end{aligned}
$$

where

$$
\lambda(x)=\frac{6 x^{2}-12 x+5}{5}, \quad \rho(x)=\frac{2 x^{2}-4 \ln x-3}{8} .
$$

The function $Y$ satisfies assumptions (4.1) with $C_{0}=1$ and $C_{1}=n(n-1)\left(T^{v}+1\right)$. The inclusion $f \in L_{x}^{2}(Q)$ holds, and we can easily verify that the function

$$
\theta(x, t)=\frac{6 x^{2}-12 x+5}{5}(t+1)+\frac{2 x^{2}-4 \ln x-3}{8} t^{2}
$$

satisfies the fractional differential equation in (2.1) and the initial and boundary conditions with the initial condition $\omega(x)=\frac{6 x^{2}-12 x+5}{5}$, which satisfies the compatibility conditions

$$
\omega_{x}(1)=0, \int_{0}^{1} x \omega d x=0
$$

Moreover, $\theta \in L_{x}^{2}(Q)$, and

$$
\partial_{t}^{\alpha} \theta=\frac{\lambda(x)}{(1-\alpha) \Gamma(1-\alpha)} t^{1-\alpha}+\frac{2 \rho(x)}{(1-\alpha)(2-\alpha) \Gamma(1-\alpha)} t^{2-\alpha} \in L_{x}^{2}(Q) .
$$

All conditions in Theorem 4.1 and Theorem 5.3 are satisfied, and the existence and uniqueness of a solution of problem (2.1) follows.

\section{Conclusion}

The existence and uniqueness of a generalized solution for a singular fractional initial boundary value problem in the Caputo sense subject to Neumann and weighted integral conditions are established. It is found that the method of energy inequalities is successfully applied to obtaining a priori estimates for the solution of the initial fractional boundary value problem as in the classical case. The obtained results will contribute to the development of the functional analysis method and enrich the existing nonextensive literature on the nonlocal fractional mixed problems in the Caputo sense.

\section{Acknowledgements}

The authors wish to thank anonymous referees for their comments and valuable suggestions.

Funding

The authors would like to extend their sincere appreciation to the Deanship of Scientific Research at King Saud University for its funding this Research group NO (RG-1435-043). 


\section{Competing interests}

The authors declare that they have no competing interests.

\section{Authors' contributions}

Both authors contributed equally to the writing of this paper. Both authors read and approved the final manuscript.

\section{Publisher's Note}

Springer Nature remains neutral with regard to jurisdictional claims in published maps and institutional affiliations.

Received: 17 January 2019 Accepted: 14 June 2019 Published online: 27 June 2019

\section{References}

1. Alikhanov, A.A.: A priori estimates for solutions of boundary value problems for fractional-order equations. Differ. Equ. 46(5), 660-666 (2010)

2. Béla, J.S., Izsák, F.: A finite difference method for fractional diffusion equations with Neumann boundary conditions. Open Math. 13, 581-600 (2015)

3. Beshtokov, M.K.H.: To boundary-value problems for degenerating pseudoparabolic equations with Gerasimov-Caputo fractional derivative. Russ. Math. 62(10), 1-14 (2018). https://doi.org/10.3103/S1066369X18100018

4. Beshtokov, M.K.H.: Local and nonlocal boundary value problems for degenerating and nondegenerating pseudoparabolic equations with a Riemann-Liouville fractional derivative. Differ. Equ. 54(6), 758-774 (2018). https://doi.org/10.1134/S0012266118060058

5. Cannon, J.R.: The One-Dimensional Heat Equation. Cambridge University Press, Cambridge (1984)

6. Caputo, M.: Elasticitae Dissipazione, Zanichelli, Bologna (1969)

7. El-Sayed, A.M.A., Gaber, M.: The Adomian decomposition method for solving partial differential equations of fractional order infinite domains. Phys. Lett. A 359, 175-182 (2006)

8. Friedman, A.: Partial Differential Equations of Parabolic Type. Prentice-Hall, Englewood Cliffs (1964)

9. Gorenflo, R., Mainardi, F., Moretti, D., Paradisi, P.: Time fractional diffusion: a discrete random walk approach. Nonlinear Dyn. 29, 129-143 (2002)

10. Huy Tuan, N., Tran Bao, N., Tatar, S.: Recovery of the solute concentration and dispersion flux in an inhomogeneous time fractional diffusion equation. J. Comput. Appl. Math. 342, 96-118 (2018)

11. Jafari, H., Daftardar-Gejji, V.: Solving linear and non-linear fractional diffusion and wave equations by Adomian decomposition. Appl. Math. Comput. 180, 488-497 (2006)

12. Kilbas, A.A., Srivastava, H.M., Trujillo, J.J.: Theory and Applications of Fractional Differential Equations. Elsevier, Amsterdam (2006)

13. Liu, F., Anh, V., Turner, I., Zhuang, P.: Time fractional advection dispersion equation. J. Appl. Math. Comput. 13, 233-245 (2003)

14. Mesloub, S.: A nonlinear nonlocal mixed problem for a second order parabolic equation. J. Math. Anal. Appl. 316 , 189-209 (2006)

15. Mesloub, S., Bouziani, A.: On a class of singular hyperbolic equations with a weighted integral condition. Int. J. Math. Math. Sci. 22(3), 511-519 (1999)

16. Podlubny, I.: Fractional Differential Equations. Academic Press, San Diego (1999)

17. Schneider, W.R., Wyss, W.: Fractional diffusion and wave equations. J. Math. Phys. 30, 34-144 (1989)

18. Wei, T., Li, Y.S.: Identifying a diffusion coefficient in a time-fractional diffusion equation. Math. Comput. Simul. 151, 77-95 (2018)

19. Widder, D.V.: The Heat Equation. Academic Press, New York (1975)

20. Xianjuan, L., Chuanju, X.: A space-time spectral method for the time fractional diffusion equation. SIAM J. Numer. Anal. 47(3), 2108-2131 (2009)

21. Yurchuk, N.I.: Mixed problem with an integral condition for certain parabolic equations. Differ. Uravn. 22(12), 2117-2126 (1986)

\section{Submit your manuscript to a SpringerOpen ${ }^{\mathcal{O}}$ journal and benefit from:}

- Convenient online submission

- Rigorous peer review

- Open access: articles freely available online

- High visibility within the field

Retaining the copyright to your article

Submit your next manuscript at $>$ springeropen.com 